\title{
The OPTX Project: Comparing the Optical and X-Ray Spectral Properties of Active Galactic Nuclei
}

\author{
Laura Trouille $^{1}$ and Amy Barger ${ }^{1}$ \\ ${ }^{1}$ Department of Astronomy, University of Wisconsin-Madison, \\ 475 N. Charter St., Madison, WI, USA \\ Email: trouille@astro.wisc.edu
}

\begin{abstract}
Using a uniformly selected and highly spectroscopically complete sample of Chandra $\mathrm{X}$-ray sources, we show that one cannot use X-ray spectral classifications and optical spectral classifications equivalently. Until a better understanding is reached for how the X-ray and optical classifications relate to the obscuration of the central engine, the use of a mixed classification scheme can only complicate the interpretation of X-ray AGN samples.
\end{abstract}

Keywords. accretion disks, galaxies: active

OPTX Sample: Trouille et al. (2008) present the X-ray, optical, infrared, and spectro-

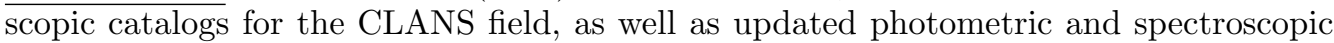
catalogs for the CLASXS and CDF-N fields. The three fields cover $\sim 1.2 \mathrm{deg}^{2}$ and contain 1789 Chandra X-ray sources. We limit this study to the $745 \mathrm{X}$-ray sources with fluxes greater than $f_{2-8 \mathrm{keV}}=3.5 \times 10^{-15} \mathrm{erg}^{-2} \mathrm{~cm}^{-1}$ and significance greater than $3 \sigma$. This " $2-8 \mathrm{keV}$ sample" is highly spectroscopically complete $\left(>80 \%\right.$ for $f_{2-8 \mathrm{keV}}>$ $10^{-14}$ erg $\mathrm{cm}^{-2} \mathrm{~s}^{-1}$ and $>60 \%$ below).

Optical Spectral Type vs. X-ray Spectral Type: Here we classify our spectroscopically identified sources into only two classes: broad-line AGNs (BLAGNs) with FWHM $>2000 \mathrm{~km}$ $\mathrm{s}^{-1}$ and non-BLAGNs with FWHM $<2000 \mathrm{~km} \mathrm{~s}^{-1}$. Under the simple unified model, the presence or absence of broad lines in the optical spectra of AGNs is due to the orientation of the observer with respect to the obscuring material near the central SMBH. $\mathrm{X}$-ray data alone have also long been used to estimate the amount of obscuration between the observer and the nuclear source through the $0.5-8 \mathrm{keV}$ spectral slope $\left(\Gamma_{\text {eff }}\right)$. Since 2-8 keV X-rays will penetrate obscuring material (except in Compton-thick AGNs) and 0.5-2 keV X-rays will not, a shallower slope may indicate an obscured source.

Trouille et al. (2009) find significant overlap of the X-ray spectral properties for the different optical spectral types, confirming results from previous studies. For example, BLAGNs are expected to be unobscured and hence X-ray soft $\left(\Gamma_{\text {eff }}>1.2\right)$, yet we find $20 \% \pm 3 \%$ have $\Gamma_{\text {eff }}<1.2$. Non-BLAGNs are expected to be obscured and hence X-ray hard $\left(\Gamma_{\text {eff }}<1.2\right)$, yet we find $33 \% \pm 4 \%$ have $\Gamma_{\text {eff }}>1.2$. These high percentages of "mismatch" between X-ray and optical spectral type require modifications to the simple unified model and provide support for alternative models for the obscuring material (Mor et al. 2009, and references therein and these proceedings).

\section{References}

Trouille, L., Barger, A. J., Cowie, L. L., Yang, Y., \& Mushotzky, R. F. 2008, ApJS, 179, 1

Trouille, L., Barger, A. J., Cowie, L. L., Yang, Y., \& Mushotzky, R. F. 2009, ApJ, 703, 2160

Mor, R., Netzer, H., \& Elitzur, M. 2009, ApJ, 705, 298 